\title{
Need for Resource Management in loT
}

\author{
Digambar Jadhav \\ Research scholar \\ Computer Engineering Department \\ D.Y.P.I.E.T., Pimpri \\ Pune, India
}

\author{
Swati Nikam \\ Computer Engineering Department \\ D.Y.P.I.E.T., Pimpri \\ Pune, India
}

\begin{abstract}
The Internet of Things (IoT) is a technical revolution which represents the future of communications and computing. In IoT many devices are connected through network and they communicate by the Internet and send data or secret information on the web. The next huge step in the IoT field is to recognize a virtual computing platform that provides access to heterogeneous group of device present in our living environment. It is nonlinear connection between resource information of smart device or client and quality of services (Qos) are built to manage the universal or limited network resource management.
\end{abstract}

\section{Keywords}

Device Management, Internet of Things, CoAP

\section{INTRODUCTION}

The next age of computing will be outside the area of desktop. RFID (Radio Frequency Identification) and sensor network technology will meet this new challenge, in which Communication system and information are invisibly embedded in the environment. The vast amount of data is processed, stored and presented efficiently seamless and easily interpretable form [1]. The term Internet of Things was first used by Kevin Ashton in 1999 in the environment of supply chain management. The definition has more wideranging applications smart-manufacturing, smart-healthcare, intelligent transportation, crowd monitoring, infrastructure health monitoring, environment monitoring, water management, smart grid etc. The main goal of IoT is making computer sense information without help of human involvement. The IoT applications can be categorized as Internet Protocol-Based and Non Internet Protocol- Based solutions. There are some IoT technology standard alliances are following 1. ZigBee 2.WAVE2M these two alliances are used for office and industry automation, and 3. WirelessHart 4. PROFIBUS used for real-time ICS etc. [2]. Internet Engineering Task Force (IETF) develops the communication protocols for resource management devices such that 6LoWPAN(Low-Power Wireless Personal area Networks) [3],Lossy Network for Routing Protocol (RPL) and low power [4], and Constrained Application Protocol (CoAP) [5], etc.IoT resource management provides a reliable and resourceful way to remotely monitor and control smart devices without consuming significant resources. It provides to universal IoT machine2machine related standardizations.

DM is a combination of network, system and application management. It includes provisioning, performance monitoring, firmware upgrades and configuration of network parameters etc. The resource management interfaces are often placed at the end of the IoT network and provides all the relevant information to the end user i.e. the device manager handling the vast amount of devices and the data [2]. Contextaware computing allows us to store context information link to sensor device data so the analysis can be done easily and more importantly. In addition, understanding context makes it easy to perform M2M communication as it is a core element in the IoT vision. When large numbers of smart devices (sensors) are deployed, and start generating data, the traditional application based approach (i.e. connect smart device (sensors) directly to applications independently and manually) becomes in-feasible. In order to address this incompetence, significant amounts of middleware solutions are introduced by researchers. Each middleware solution focuses on different aspects in the IoT, such as DM, security, privacy platform portability and context-awareness, and many more [8].

\section{RELATED TO WORK}

Authors have proposed a trivial RESTful Web service (WS) approach. This approach is used to enable device management (DM) of wsn. They have implemented IPv6 over low-power wireless personal area network (6LoWPAN)/routing protocol for low power and lossy network (RPL)/ CoAP protocols on smart devices. They suggested a CoAP-based DM solution that provides ease in accessing and managing IPv6 sensor devices. The remote access and management from an Internet device to an IPv6 smart device can be incorporated by combining IEEE 802.15.4 HTTP-CoAP proxy and connectivity into an open-platform gateway. They have considered some issues like Real-time management for resource constrained sensor networks; dynamic registration, bootstrap and management for a large-scale deployment with smart devices coming in and out and altering their functionalities and characteristics [2].

Authors have proposed the implementation of existing IP based network management protocol on resource constrained devices. In an implementation, SNMP (Simple network management Protocol) provides resourceful use of resources on constrained devices and security is also provided. It is compared with NETCONF implementation which requires more time for processing requests, not allow for any security expenses. Adding security to NETCONF increases overhead to session start up time. The key challenges are related to message framing issues, session establishment and maintenance issues, security issues. The protocols using small application layer messages that fit into one IPv6 packet are much better suitable for memory constrained devices [10].

Authors have focused on the importance of middleware for IoT. Fundamental functional blocks are projected for middleware system. No standard middleware is available which can be applied across all possible smart environmentslike smart city, smart home, including RFID domain, and can be modified as per the domain specific requirements. The study says that in order to resolve scalability issues, IPV6 is projected but it is not completely achieved. Even support for processing and context is not accomplished fully. Open issue also includes support of managing of data volumes and semantic modeling. Research can be done in making generic middleware system that can be applied to all domains by reuse 
all the functional components. Also it can be added as add-on to the middleware system [9].

Authors have proposed different approaches for deploying interoperable Simple Object Access Protocol (SOAP)-based web services this is directly on the nodes and not using gateways. This policy provides for easy integration with legacy IT systems and supports heterogeneity at the lowest level. Two fold analysis of the related transparency, which is the main challenge. Quantification of resource spending as well as techniques to mitigate it is presented. Here one challenge found that overhead related to SOAP message processing is very small compare to message transmission [11].

In order to achieve resourceful embedded web services are introduce: a fresh approach to a web application transfer protocol and resourceful payload encoding two key activities required. Here, analyzes the most promising payload encoding techniques and introduces the new IETF Constrained RESTful Environments (CoRE) standardization activity. Here, applied REST for the manipulation of resources using HTTP and RPC style interactions using Simple Object Access Protocol (SOAP). Future challenges like is growing internet scale in billions to trillions of nodes and interaction between models change as autonomous actions. The most important challenges are routing scalability, naming and identification, security, privacy, and the manageability network [12].

\section{PROTOCOL DESCRIPITION IN RESOURCE CONSTRAINED NETWORKS}

The Internet Engineering Task Force (IETF) introduced following protocol Fig.1.used in resource constrained networks [15]. These protocols used for communication between user and smart objet in resource management.

\begin{tabular}{|c|c|}
\multicolumn{1}{c|}{ Layers } & Protocol \\
\hline Application Layer & CoAP \\
\hline Transport Layer & TCP/UDP \\
\hline Network Layer & RPL \\
\hline Adaption Layer & 6LoWPAN \\
\hline MAC Layer & IEEE 802.15.4 \\
\hline Physical Layer & IEEE 802.15.4 \\
\hline
\end{tabular}

Figure.1.Protocol description for resource management network [2].

1. CoAP

- It is M2M web protocol.

- $\quad$ REST architecture.

- It is caching capabilities and simple proxy

- It is asynchronous transaction support.

- Low header overhead and parsing complexity.

- Reliable unicast and best-effort multicast support.

2. TCP/UDP

TCP-

- It is a connection oriented protocol.

- It is high reliability, transmission time is less critical.

- It is rearrange packets.

UDP-
- It is alternative for TCP

- It is specially tune for real time performance

- It is connectionless protocol.

3. RPL

- UDP is used to message transfer.

- Low power and Lossy Networks (LLNs) consisting largely of constrained nodes.

- Lossy and unstable links, typically supporting low data rates, relatively low packet delivery rates.

- Traffic patterns are not simply point-to-point, but in many cases point-to-multipoint or multipoint-topoint.

- Potentially comprising up to thousands of nodes.

4. 6LoWPAN

- This protocol is an adaptation layer allowing transporting IPv6 packets over 802.15.4 links.

- It uses 802.15.4 in unslotted CA/CSMA mode.

- Reassembly/ Fragmentation of IPv6 packets.

- Compression of UDP/ICMP and IPv6 headers.

- Mesh routing support.

5. IEEE 802.15.4

- It is specifies both MAC layer and physical layer.

- It is radio technology for low data rate and low power.

- It is limited range.

- Maximum Data Rate $250 \mathrm{~kb} / \mathrm{s}$ and maximum packet size 127 bytes

\section{COMMUNICATION METHODS OF RESOURCE MANAGEMENT}

In resource management system two communication method developed CoAP-Based and RESETfulBased.These methods are explained following [2].

1. CoAP-BASED Resource Management using WSN It is communication method of IoT client and a smart device, specially the device components interface to IoT client. There are five major functions of this communication method. These functions are described the following.

1) Register: It is a main function in resource management to allow register or deregister smart object and maintain the registration information of smart object.

2) Provisioning: This function synchronize and initialize information of a smart device with a server.

3) Management services: Smart device is linked to remote server, a no. of important management services should take in accuse to maintain IoT services, status inquiry, parameter configuration and connection identify, and remote control.

4) Observing: It is the unique feature of CoAP to allow sensor devices to "examine" resources.

5) Application data transmission: It is application data that can be collect and deliver to IoT clients, or any other application protocols above CoAP.

The CoAP is based on the replace messages are elated over User Datagram Protocol (UDP).Reliability over the UDP transport is provided by the built-in retransmission mechanism of CoAP, e.g., CON message defined by the Type field in the header. It could also be used over other transport protocols such as TCP or SMS. CoAP messages are determined in a plain binary format. The message format 
starts with a fixed-size 4-byte header. This is followed by a variable-length token value which can be between 0 and 8 bytes long. [2].

\section{RESTful Resource Management}

This type of communication method in resource management is access from Internet application to smart devices, especially for those of internet users who cannot use CoAP, it integrate IEEE 802.15.4 connectivity into an open-platform gateway and port the HTTP-CoAP (HC) proxy to the OpenWrt, the operation system of the gateway, to access from an ordinary IP terminal to an IPv6 sensor device.

The following layers described the resource management in RESTful.

1) libcoap Layer: It defines message structure to apply the CoAP messages layer based on UDP.

2) CoAP Request/Response Layer: It is transmit CoAP call for in the form of CoAP message through the messages and generate CoAP response based on received CoAP messages.

3) HTTP-CoAP Mapping Layer: It is implement map from HTTP request to CoAP request. It converting a HTTP request to CoAP request, the HC proxy needs to convert the HTTP request method, URI, reader/option and payload, respectively. If a proxy encounters an error, it has to generate corresponding error response.

\section{IoT MIDDLEWARE ARECHITECTURE}

The IoT middleware layers are device abstraction responsible for providing interoperation, interface protocols, semantics associated with devices, resolving the syntax and management module is the central parts which perform the device discovery, management and context discovery [9].

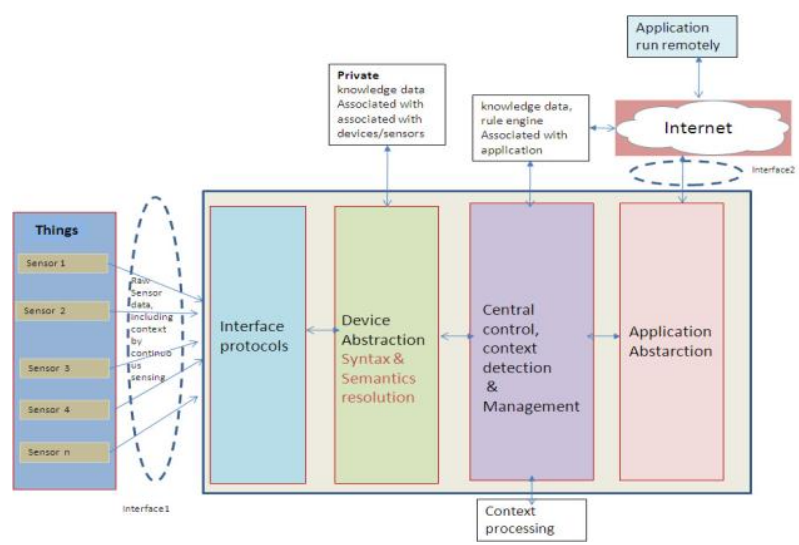

Figure.2. Functional Components of IoT-middleware [9].

1. Interoperation: It distributes information and uses the same across different domains of applications using different communication interfaces. It classifies three different categories like syntactic, network and semantics. Network interoperation defines protocols for exchange information among the variety of things across special communication networks, without consider content of information.

2. Context Detection: it can realize context processing and context detection. Context detection is collect data and identifies the factor. Context processing extracts the data, processes it and performs or takes decision.

3. Device Discovery Management: Enables any device in the IoT network to identify all nearest device and make its incidence known to each neighbor in the network.

4. Security and Privacy: It is responsible for authenticity, secrecy and non-repudiation in network [9].

\section{NEED FOR RESOURCE MANAGEMENT}

The IoT is a relation between real world and virtual world. IoT are millions of smart object connected to internet. In resource management are managing these millions of object in single application.

There is various issues related to resource management in IoT.

1. The need for resource management is a real time network monitoring and control network.

2. Real time fault reporting is an important characteristic of resource management.

3. Detecting the possible anomaly is an important point for the IoT resource management to ensure the reliability of the data.

4. Increasing the smartness of nodes to increase the efficiency of the constrained networks.

5. To decrease the power consumption.

6. To track the all devices connected to internet.

The main goal of resource management is better utilize of the open resources and raising the Qos (quality of the services) accessible to the people, while reducing the expenses of the public administration.

\section{CONCLUSION}

We have discussed the resource management communication framework such as CoAP-based and Restful method. We have also presented the middleware architecture in IoT. The current state of the middleware for IoT explores different approach to support some functionality to control in IoT domain. But no one cover the complete set of functionality to meet the requirement of IoT middleware as analyzed here for any smart environment. We have discussed the features of resource management such as publish/subscribe, Aggregation, prioritization, Anomaly detection, error reporting.

\section{REFERENCES}

[1] Gubbi, Jayavardhana, et al. "Internet of Things (IoT): A vision, architectural elements, and future directions." Future Generation Computer Systems Vol.29.Issue 7, Year 2013,Pages 1645-1660.

[2] Sheng, Zhengguo, et al. "Lightweight management of resource constrained sensor devices in internet-ofthings." IEEE internet of things journal Vol. 2,Issue. 5, Year 2015, Pages 402-411.

[3] G. Montenegro, N. Kushalnagar, J. Hui, and D. Culler, "Transmission of IPv6 packets over IEEE 802.15.4 networks," Internet Engineering Task Force, RFC 4944, 2007.

[4] T. Winter et al., "RPL: IPv6 routing protocol for lowpower and lossy networks," IETF, RFC 6550, 2012 
[5] Z. Shelby, K. Hartke, and C. Bormann, (2014 Jun.) Constrained Application Protocol (CoAP), Internet Draft [Online].Available:http://datatracker.ietf.org/wg/core/cha rter

[6] Song, J., Kunz, A., Schmidt, M., \& Szczytowski, P. Connecting and managing $\mathrm{m} 2 \mathrm{~m}$ devices in the future internet. Mobile Networks and Applications, Vol. 19, Issue 1, Year 2014, Pages 4-17.

[7] Swetina, Jorg, et al. "Toward a standardized common $\mathrm{m} 2 \mathrm{~m}$ service layer platform: Introduction to onem $2 \mathrm{~m}$." Wireless Communications, IEEE Vol.21, Issue.3, Year 2014,Pages 20-26.

[8] Perera et al.: et al. " Contextaware computing for the internet of things:A survey." Wireless Communications, IEEE Vol.16, Issue.1,Year 2014,Pages 414-454.

[9] Bandyopadhyay, S., Sengupta, M., Maiti, S., \& Dutta, S. Role of middleware for internet of things: A study." International Journal of Computer Science \& Engineering Survey (IJCSES),Vol. 2,Issue 3, Year 2011 Pages 94-105.

[10] Anuj Sehgal, et.al. "Management of Resource Constrained Devices in the Internet of Things" IEEE Communications Magazine,Vol. 50 ,Issue 12 ,Year 2012 ,Pages 144-149.

[11] Rumen Kyusakov et.al. "Integration of Wireless Sensor and Actuator Nodes With IT Infrastructure Using Service-Oriented Architecture"IEEE Transactions on industrial informatics, Vol. 9, Issue. 1, Year 2013,Pages $43-51$

[12] Zach Shelby,et. al."Embedded Web Services" Wireless Communications IEEE Vol.17,Issue 6,Pages 52-57.

[13] Anuj Sehgal, et.al. "Management of Resource Constrained Devices in the Internet of Things" IEEE
Communications Magazine,Vol. 50 ,Issue 12 ,Year 2012 ,Pages 144-149.

[14] A. Hergenroeder, J. Wilke, and D. Meier, "Distributed energy measurements in WSN testbeds with a sensor node management device (SNMD)," in Proc. 23rd Int. Conf. Archit. Comput. Syst. (ARCS), Year 2010, Pages 1-7.

[15] Sheng, Zhengguo, et al. "A survey on the IETF protocol suite for the internet of things: Standards, challenges, and opportunities." Wireless Communications, IEEE Vol.20, Issue 6 Year 2013, Pages 91-98.

[16] Atzori, L., Iera, A., Morabito, G.: The Internet of Things: A Survey. In: Computer Networks,vol. 54, issue 15, Year 2010, Pages. 2787-2805.

[17] T. Cucinotta et. al.,"A real-time service-oriented architecture for industrial automation," IEEE Transactions on industrial informatics, Vol.5, Issue.3, Year 2009, Pages 267-277.

[18] J. Stankovic et. al.,"Research directions for the Internet of Things," IEEEInternet Things Journal., Vol. 1, Issue. 1, Year 2014, Pages 3-9.

[19] J. Song, A. Kunz, M. Schmidt, et. al, "Connecting and managing M2M devices in the future Internet," in Mobile Networks and Applications, New York, NY, USA: Springer, Vol. 19, Year 2014, Pages 4-17.

[20] http://www.rfidjournal.com/articles/view?4986

[21] http://www.sap.com/platform/netweaver/components/ind ex.epx

[22] http://www.opengeospatial.org/standards/sensorml

[23] http://wendang.baidu.com/view/ad7040a1b0717fd5360c dc8a.html 